\title{
A configuration optimizing method in robotic machining
}

\begin{abstract}
Industrial robots have been wildly used in machining applications due to its high flexibility and low-cost. However, the relatively low stiffness of robots seriously influences their machining accuracy and quality. Based on the redundant degree of freedom and the robotic static stiffness model, machining configuration of six-revolute serial robots could be optimized off-line to improve kinematics performance and stiffness performance. This paper addresses a configuration optimizing method to off-line optimize configuration which far away from singularity and joint-limits meanwhile achieve the optimum stiffness.
\end{abstract}

Keywords: Industrial robots; Redundant degree of freedom; Singularity avoidance; Joint limits avoidance; Stiffness performance
Volume 3 Issue 2 - 2017

\author{
Jiachen Jiao,Wenhe Liao,Wei Tian,, Lin Zhang, \\ Shuang Long Liu
}

Nanjing University of Aeronautics and Astronautics, China

Correspondence: Wei Tian, Nanjing University of Aeronautics and Astronautics, China, Tel I385I66233I, Fax 84891836,

Email tw_nj@nuaa.edu.cn

Received: August 31, 2017| Published: October 10, 2017

\section{Introduction}

Industrial robots have been more and more used in industrial field, because of the advantages of high flexibility, high automation level and low-cost. As robotic technology development and progress unceasingly, higher performance requirements are proposed for the utility of industrial robot. Since six-axis robot is redundant in the machining process, each position of Tool Center Point suits a series of robot configurations. The redundancy problem including how to utilize the redundancy to optimize the posture far away from singularity and joint-limits posture, concurrently guarantee and improve the machining quality. A new performance index is addressed for the combined requirements of singularity and jointlimits avoidance, and it optimizes the robot configurations at joint displacement level. In addition, the low stiffness property is a main limitation to the robot application in the accurate machining. The robotic stiffness performance relates to configuration directly. This paper addresses a stiffness modeling method for machining tasks. A quantitative evaluation index is proposed to stiffness performance and it can be used to optimize configurations further on the basis of kinematics performance optimization.

\section{Performance index for joint-limits and singularity avoidance}

Manipulability is a comprehensive measurement of ability to move in all directions, is an effective way to achieve manipulator designing, task planning and fast recovery from singular positions. Because of the lower computation and more intuitive distinguish to singular configuration, we choose manipulability to measure the distance to singularity, which formulate as ù $=\sqrt{\operatorname{det}\left(\mathrm{JJ}^{\mathrm{T}}\right)} \cdot{ }^{1}$ In order to realize joint-limits avoidance, we need to keep each joint far away from its limited position. The performance index which could be written as below is defined to keep the robot joint as close to midjoint position á as possible to prevent reaching joint-limits even collision with obstacles. The performance index can be formulated as $\mathbf{H}()=\sum_{\mathrm{i}=1}^{\mathrm{n}}\left(\frac{\grave{\mathrm{e}}_{\mathrm{i}}-\dot{\mathrm{a}}_{\mathrm{i}}}{\grave{\mathrm{e}}_{\text {imax }}-\mathrm{a}_{\mathrm{i}}}\right)^{2}$. The singularity avoidance index ranges from zero to a limited maximum positive number; zero is happened at the singularity posture. However, the value of joint-limits avoidance index at the mid-joint position is zero, and the value goes up to 1 when reach the limit. These two indexes are incomparable. So structure changes of these two indices are necessary to receive a combined performance index which obeys the principle: the value of the new index can avoidant the singularity configuration and joint-limits configuration simultaneously. ${ }^{2}$ Based on the describe, the combined performance index is acquired:

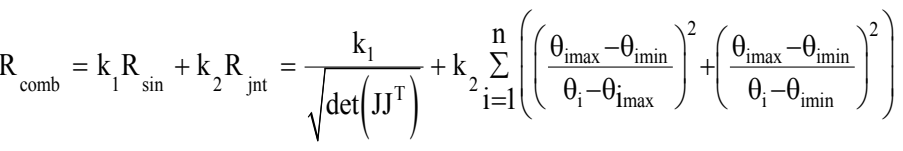

Evaluation method of robot stiffness

\section{performance}

There are three elements influents robotic stiffness:

\section{a. Material and structure}

b. Actuator and transmission system

c. Configuration of robot

Only the third one could be adjusted, so it is regarded as the only factor influences the robotic stiffness, three assumptions are raised. First, the end effector is considered as a rigid body, all deformations attributes to the weak stiffness of robot. Next, the elastic deformations cause all the robotic displacements. Finally, the robot keeps stable during process. By using conservative congruence transformation, Chen and Kao proposed a method to define the relationship of stiffness matrix between joint and Cartesian space, whose formula can be simplified as: $\mathrm{K}=\mathrm{J}^{-\mathrm{T}} \mathrm{K}_{\theta} \mathrm{J}^{-1} \quad{ }^{3,4}$ The formula is called traditional model of static stiffness, $\mathrm{J}$ changes follow the robot configuration, so robot configuration has a great influence on robotic stiffness performance.

During machining process, torque has negligible influence on the translational displacement of end effector. There only the relation between force and translational displacement is studied: $\mathrm{f}=\mathrm{K}_{\mathrm{fd}} \mathrm{d}$. Suppose $\mathrm{f}$ as a unity force and $\mathrm{f}^{\mathrm{T}} \mathrm{f}=\mathrm{d}^{\mathrm{T}} \mathrm{K}_{\mathrm{fd}}^{\mathrm{T}} \mathrm{K}_{\mathrm{fd}} \mathrm{d}=1$. The formula describes an ellipsoid which changes follow the configuration, denoted as stiffness ellipsoid. The eigenvectors of $\mathrm{K}_{\mathrm{fd}}^{\mathrm{T}} \mathrm{K}_{\text {fd }}$ are the directions of ellipsoid's principal axes. The values of ellipsoid's semi axis $\lambda_{1}, \lambda_{2}, \lambda_{3}$ are singular values of the matrix. $\lambda_{3}$ Is the 
shortest axial length, in other words, the lowest stiffness is obtained in this direction. Therefore, it could be seen as the integrated rigid index in the workspace. In view of the stiffness in all directions contribute to robotic stiffness in a certain configuration; it is feasible that deem all-around stiffness is related to the volume of ellipsoid: $\mathrm{V}=\frac{4}{3} \pi \lambda_{1} \lambda_{2} \lambda_{3}=\frac{4}{3} \pi \operatorname{det}\left(\mathrm{K}_{\mathrm{fd}}^{\mathrm{T}} \mathrm{K}_{\mathrm{fd}}\right)$. In order to have a similar order of magnitude and the unit of stiffness, the all-round stiffness index could be defined as: $\mathrm{k}_{\mathrm{a}}=\sqrt[6]{\operatorname{det}\left(\mathrm{K}_{\mathrm{fd}}^{\mathrm{T}} \mathrm{K}_{\mathrm{fd}}\right)}$.

\section{Robotic configuration optimization method}

There are a series of robotic configurations satisfied the position and direction of each machining position due to the redundancy of the sixrevolute robot. Thus, method of optimizing machining configuration could be seen as redundancy resolution schemes. The configuration optimization is conducted at the level of joint displacement using mathematical optimization method. The steps as follow: At first, read processing information and abstract location point and normal vector messages of target positions. Secondly, the initial joint values are obtained by the inverse kinematics method. ${ }^{5}$ The value of stiffness performance index is calculated by the initial configuration. Next, rotate the end effector around the machining axis with a series of angles which gradual increases and generate new candidates of the robotic configuration; then, filter the configuration by the limit of joint value's usable range which obtained through the kinematics performance index. If the configuration can't meet the conditions, go to the next one. If not, calculate the stiffness performance index and compare the value with $\mathrm{k}$ and refresh $\mathrm{k}$ by the bigger one; Finally, repeat above steps, obtain the optimal rotation angle and the optimal configuration.

\section{Conclusion}

Based on the redundancy of degree of 6-serial robot, a kinematics index is addressed to pretend the singularity and joint-limits configurations. And a stiffness performance index is come up with to evaluate the robot all-around stiffness through the study that the different postures' influence. Combine the kinematic performance and stiffness performance indexes; a configuration optimizing method is put forward. In this way, the accuracy, quality and efficiency of machining task can be effectively improved.

\section{Acknowledgments}

The research was supported by the National Natural Science Foundation of China (NO.51575273).

\section{Conflict of interest}

No financial interest or any conflict of interest exists.

\section{References}

1. Yoshikawa T. Manipulability of robotic mechanisms. Int $j$ robot res. $1985 ; 4(2): 3-9$.

2. Weidong Zhu, Weiwei Qu, Lianghong Cao, et al. An off-line programming system for robotic drilling in aerospace manufacturing. International Journal of Advanced Manufacturing Technology. 2013;68(9-12):25352545 .

3. Chen S. Conservative congruence transformation for joint and Cartesian stiffness matrices of robotic hands and fingers. The International Journal of Robotics Research. 2000;19(9):835-847.

4. Alici G, Shirinzadeh B. Enhanced stiffness modeling, identification and characterization for robot manipulators. IEEE Transactions on Robotics. 2005;21(4):554-564.

5. Paul RP, Shimano B. Kinematic control equations for simple manipulators. In: Decision and Control Including the Symposium on Adaptive Processes. 1978 IEEE Conference. 1979. p. 1398-1406. 